\title{
Modelos de regressão para predição do peso da raça Canindé através de medidas morfométricas
}

\author{
Conrado, V.D.C.; Arandas, J.K.G. e Ribeiro, M.N. ${ }^{@}$
}

Departamento de Zootecnia. Universidade Federal Rural de Pernambuco. Recife. PE. Brasil.

\section{PaLAVRAS CHAVE ADICIONAIS}

Equação.

Medidas corporais.

Sistema extensivo.

\section{ADDITIONAL KEYWORDS}

Body measurements.

Equation.

Extensive system.

\section{INFORMACIÓN}

\section{Cronología del artículo.}

Recibido/Received: 11.7.2014

Aceptado/Accepted: 9.8.2015

On-line: 16.9.2015

Correspondencia a los autores/Contact e-mail:

ribeiromn1@hotmail.com

\section{RESUMO}

O presente trabalho teve como objetivo utilizar equações de predição para estimar o peso vivo de caprinos da raça Canindé mediante medidas morfométricas de natureza quantitativa. Foram avaliadas 347 fêmeas adultas da raça Canindé, provenientes de diferentes estados na região Nordeste do Brasil. Os animais foram pesados e, após as pesagens, com o auxilio de uma fita métrica foram tomadas medidas de altura da cernelha (AC), comprimento do corpo (CC) e perímetro torácico (PT). Foram observadas correlações significativas entre o peso e todas as variáveis avaliadas. A variável melhor correlacionada com o peso foi o PT $(0,69)$. Os modelos polinomiais foram os que apresentaram o melhor ajuste, com maior precisão para aquele com três variáveis. A metodologia utilizada mostrou-se adequada para predizer o peso de fêmeas adultas da raça Canindé para as condições em que o trabalho foi conduzido.

\section{Regression models to predict the weight of Caninde goat breed through morphometric measures}

\section{SUMMARY}

This study aimed to identify equations to estimate the live weight of Caninde goat breed by quantitative morphometric measurements. It was evaluated 347 adult females come from different states of Northeast region, Brazil. Each animal was weighed and after that it was taken measures of height at withers (HW), body length (BL) and thoracic perimeter (TP). Significant correlations were observed between the weight and all variables. The variable best correlated with the weight was the PT (0.69). The polynomial models presented the best fit with higher accuracy to one with three variables. The methodology was effective to predict the weight of adult females in the conditions under which the work was conducted.

\section{INTRODUÇÃO}

A caprinocultura é uma atividade de alta potencialidade tanto social como econômica para as populações de média e baixa renda do Nordeste brasileiro, onde se encontra $90 \%$ do rebanho, destacando-se a predominância de animais criados em sistema extensivo (IBGE, 2009). Esse tipo de sistema não suporta animais altamente especializados, sendo comum a utilização de caprinos locais, como os da raça Canindé, considerada uma das principais raças locais da região Nordeste do Brasil, sendo caracterizados como animais altamente adaptados e de múltiplas funções (leite, carne e pele). A produção média diária de leite para esta raça é de 0,760 $\mathrm{kg}$, sendo essa acima da média dos caprinos locais do Nordeste do Brasil (Santana, 2000).

Apesar de sua importância econômica, social e cultural, a raça Canindé vem sofrendo processo de diluição genética e, por isso, atualmente, é uma raça considerada ameaçada (Lima et al., 2007), apresentando rebanhos com tamanho efetivo inferior ao valor mínimo recomendado pelo FAO (1998), que é de 50. Uma das principais causas dessa ameaça são os cruzamentos desordenados com raças exóticas (Ribeiro et al., 2004a) associado ao desconhecimento do seu potencial produtivo.

O conhecimento acerca dos parâmetros fenotípicos, que compreende as características produtivas e reprodutivas dos animais é importante de modo a auxiliar na definição de programas de conservação para as raças locais em geral, informações que devem ser devidamente acompanhados para a garantia do sucesso das criações e manutenção das raças.

Na espécie caprina, alguns fatores afetam o seu rendimento de carcaça, como idade, peso ao abate, 


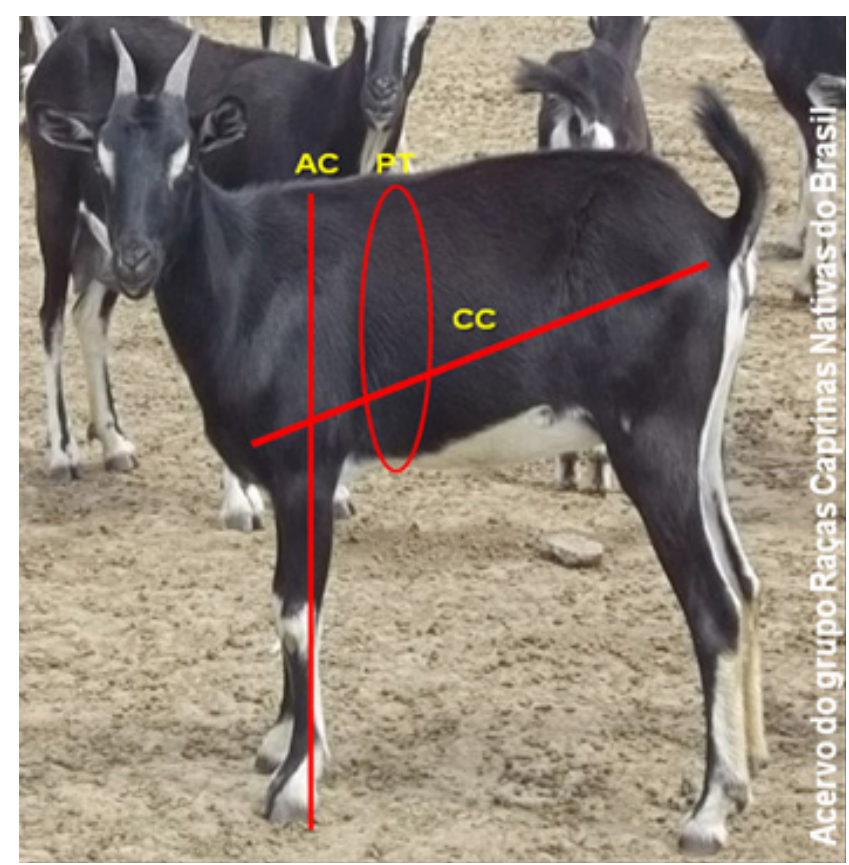

Figura 1. Localização das medidas morfométricas realizadas nos animais estudados (Location of morphometric measurements done in studied animals).

sexo e sistema de criação (Mattos et al., 2006). O peso é um parâmetro fundamental para a avaliação do desenvolvimento dos animais, no entanto o uso limitado de novas tecnologias e o baixo investimento em infraestrutura é comum a maioria dos pequenos produtores rurais. Tal fato impossibilita a pesagem periódica dos animais, dificultando o controle ponderal e a comercialização dos animais baseada no peso vivo.

A utilização de modelos de regressão para predizer o peso dos animais a partir de medidas morfométricas surgiu como uma possível solução para essa problemática. Esse tipo de estudo ganha maior relevância nas raças caprinas criadas em sistemas extensivos, onde o controle zootecnico é precario ou inexistente, notadamente em criações de raças nativas.

Urbano et al. (2006) cita a importância da estimação do peso vivo através de medidas corporais no controle zootécnico, em casos nos quais não há disponibilidade de balança.

A utilização de equações de regressão a partir de medidas morfométricas pode ser uma ferramenta importante para predição do peso vivo dos animais em todas as idades (Osório et al., 1996). Diante disso, o objetivo desse trabalho foi utilizar equações de predição para estimar o peso de caprinos adultos da raça Canindé criados em sistema extensivo.

\section{MATERIAL E MÉTODOS}

Os dados foram obtidos de 347 fêmeas adultas (acima de dois anos de idade) da raça Canindé, provenientes de diferentes estados na região Nordeste do Brasil e criadas em sistema extensivo de criação. Os animais/ estados foram pesados em balança e, após as pesagens, com o auxílio de uma fita métrica foram medidas a altura da cernelha $(\mathrm{AC})$ que corresponde à medida
Tabela I. Médias e respectivos desvios-padrão $(\mu \pm D P)$ do peso, medidas morfométricas e coeficientes de variação $(\mathrm{CV})$ em fêmeas da raça Canindé (Location of morphometric measurements in animal studies).

\begin{tabular}{lcr}
\hline Variáveis & $\mu \pm \mathrm{DP}$ & $\mathrm{CV}(\%)$ \\
\hline Peso adulto & $30,11 \pm 4,93$ & 16,37 \\
Perímetro torácico & $74,66 \pm 4,42$ & 5,92 \\
Comprimento do corpo & $68,93 \pm 4,25$ & 6,16 \\
Altura da cernelha & $59,45 \pm 3,61$ & 6,06 \\
\hline
\end{tabular}

$\mathrm{N}: 347$ animais.

realizada a partir do solo até o ponto mais culminante da região interescapular; comprimento do corpo (CC), medida da articulação da escápula-humeral até a extremidade posterior do ísquio e perímetro torácico (PT), totalizando 347 amostras. As pesagens e as medidas foram coletadas durante uma visita a cada propriedade/estado. Essa medida é feita a partir do ponto de declividade da região interescapular, envolvendo toda região torácica (figura 1).

As características morfométricas e o peso dos animais foram submetidos à análise descritiva simples e análise de correlação de Pearson através dos procedimentos Proc Means e Proc Corr do SAS (1999), respectivamente.

A obtenção das equações de predição e teste dos diferentes modelos (lineares, quadrática e polinomial) de regressão foi realizada pelo procedimento Proc Reg do SAS (1999), tendo como variável dependente o peso corporal dos animais. O melhor modelo indicado para estimar o peso observado dos animais foi escolhido com base no maior coeficiente de determinação $\left(R^{2}\right)$.

\section{RESULTADOS E DISCUSSÃO}

As médias de peso adulto, perímetro torácico (PT), comprimento do corpo (CC), altura da cernelha (AC) e seus respectivos desvios-padrão para fêmeas adultas da raça Canindé encontram-se na tabela I. Os coeficientes de variação das características avaliadas variaram de 5,92 a 16,37\%, sendo valores considerados muito bons para dados obtidos em condições de campo. A média de peso $(30,11 \pm 4,93)$ para fêmeas adultas da raça Canindé foi inferior ao peso indicado por Ribeiro et al. (2004b) para a raça. Se tratando da mesma raça essas diferenças podem estar relacionadas com os efeitos ambientais como época de menor ocorrência de chuvas que é propicio a menor disponibilidade de forragem além da influência do ano e mês de coletada de dados.

$\mathrm{Na}$ tabela II, encontram-se as correlações de Pearson entre as variáveis avaliadas para os animais estudados. Observam-se correlações médias e significativas $(\mathrm{p}<0,01 \%)$ entre todas as variáveis avaliadas, as quais variaram de $42 \%$ a $69 \%$. A variável melhor correlacionada com o peso foi o PT, o que corrobora com Ribeiro et al. (2004b) em estudos com caprinos da raça Canindé e Moxotó e Tadesse et al. (2012) com caprinos nativos na Etiópia encontraram alta correlação entre o PT e o peso vivo dos animais. O perímetro torácico é importante indicador do peso vivo, rendimento da carcaça, 
Tabela II. Correlações de Pearson entre as variáveis avaliadas em fêmas adultas da raça Canindé (Pearson correlations among the variables in adult female of Caninde breed).

\begin{tabular}{lllll}
\hline & AC & PT & PA & CC \\
\hline AC & 1,00 & $0,44^{* *}$ & $0,47^{* *}$ & $0,42^{*}$ \\
PT & & 1,00 & $0,69^{* *}$ & $0,54^{* *}$ \\
PA & & & 1,00 & $0,53^{* *}$ \\
CC & & & & 1,00 \\
\hline
\end{tabular}

${ }^{* *}: p<(0,01) . A C=$ altura da cernelha; $P T=$ perímetro torácica; $P A=$ peso adulto; $\mathrm{CC}=$ comprimento do corpo.

Tabela III. Equações de predição de peso adulto (PA) de fêmas de caprinos da raça Canindé(Prediction equations for adult weight (PA) of adult female of Caninde goats).

\begin{tabular}{llc}
\hline Modelo & Equações & $\mathrm{R}^{2}$ \\
\hline Linear simples & $\mathrm{PA}=-27,32+0,77(\mathrm{PT})$ & 0,48 \\
Quadrática & $\mathrm{PA}=8,19-0,18(\mathrm{PT})+0,01\left(\mathrm{PT}^{2}\right)$ & 0,48 \\
Polinomial(1) & $\mathrm{PA}=-35,16+0,63(\mathrm{PT})+0,26(\mathrm{CC})$ & 0,51 \\
Polinomial(2) & $\mathrm{PA}=-41,32+0,57(\mathrm{PT})+0,21(\mathrm{CC})+0,24(\mathrm{AC})$ & 0,53 \\
\hline
\end{tabular}

$\mathrm{R}^{2}=$ Coeficiente de determinação.

capacidade digestiva e respiratória dos animais (Santana et al., 2001), sendo bastante útil como indicador do desempenho do animal.

Valdez et al. (1982), trabalhando com caprinos de diferentes grupos raciais e sexos, e com ampla variação de peso e idade, também observaram que o perímetro torácico foi a melhor medida para estimar o peso vivo. Mohamed e Amin (1996) recomendaram a utilização do perímetro torácico para determinar o peso vivo de caprinos e ovinos de diferentes pesos e categorias zootécnicas, Mahieu et al. (2011) e Randrianariveloseheno et al. (2015) destacaram a importância do perímetro torácico como medida confiável para estimar o peso de caprinos nativos em Guadalupe e Madagascar, respectivamente em contrapartida, Osório et al. (1999), ao trabalharem com cordeiros Corriedale, com 9,5 meses de idade, observaram que o perímetro torácico foi, na ordem, a quarta variável melhor correlacionada.

Na tabela III, encontram-se as equações de predição de peso de caprinos da raça Canindé criados em sistema extensivo. Observa-se através do coeficiente de determinação $\left(R^{2}\right)$ que os modelos linear simples e quadrática apresentaram ajustes semelhantes. Para os modelos polinomiais foi obsevado pequena diferença nos ajustes com maior valor para o modelo que incluiu três variáveis. Comparativamente, todos os modelos estudados poderiam ser usados para predição do peso adulto com margem de erro semelhante. Resende et al. (2001) citando Valdez et al. (1982) afirmaram que a utilização de equações de regressão em função de várias medidas biométricas produzem pequeno aumento na precisão das estimativas obtidas, o que corrobora com os resultados obtidos no presente estudo. Este fato pode ser verificado pelo ganho em precisão conseguido entre os modelo polinomial 1 e 2, no qual a inclusão de mais uma variável promoveu aumento no $\mathrm{R}^{2} \mathrm{de}$ apenas $3 \%$.

\section{CONCLUSÕES}

Os modelos avaliados são capazes de predizer o peso corporal de fêmeas adultas da raça Canindé nas mesmas condições utilizadas neste estudo e com a mesma margem de precisão, com destaque para os modelos polinomiais que aparentaram um melhor ajuste em comparação aos demais modelos. As equações de predição são uma ferramenta barata e de fácil utilização para estimar o peso de vivo de caprinos, no entanto as equações acima estimadas foram desenvolvidas para caprinos da raça Canindé e necessitam de validação para outras raças.

\section{BIBLIOGRAFIA}

Food and Agriculture Organization - FAO. 1998. Secondary guidelines for development of national farm animal genetic resources management plans. Management of small populations at risk. In:Woolliams, J.A.; Gwaze, G.P.; Meuwissen, T.H.E. et al. (Eds.). Food and Agriculture Organization of the United Nations.

IBGE. Instituto Brasileiro de Geografia e Estatística. 2009. Produção da pecuária mundial. Rio de Janeiro. v. 37: 1-55.

Lima, P.J.S.; Souza, D.L.; Pereira, G.F.; Torreão, J.N.C.; Moura, J.F.P.; Gomes, J.T.; Lopez Acosta, J.M.; Rey Sanz, S.; M.N. Ribeiro e Pimenta Filho, E.C. 2007. Gestão genética de raças caprinas locais no Estado da Paraíba. Arch Zootec, 56: 623-626.

Mahieu, M.; Navès, M. and Arquet, R. 2011 . Predicting the body mass of goats from body measurements. Livest Res Rural Develop, 23(9) (03/09/2012).

Mattos, C.W.; Carvalho, F.F.R. e Dutra Junior, W.M. 2006. Características de carcaça e dos componentes não-carcaça de cabritos Moxotó e Canindé submetidos a dois níveis de alimentação. Rev Bras Zootecn, 35: 2124-2134.

Mohamed, I.D. and Amin, J.D. 1996. Estimating body weight from morphometric measurements of Sahell (Borno Withe) goats. Small Ruminant Res, 24:1-5.

Osório, J.C.S.; Vaz, C.M.S.L.; Jardim, P.; Pimentel, M. e Loguercio, A. 1996. Componentes do peso vivo na raça Crioula. Congresso Brasileiro de Medicina Veterinária, 25. Anais... CONBRAVET. Gramado-RS. 266 pp.

Osório, J.C.; Osório, M.T.; Vaz, C.M.; Mendonça, M.G.; Gonçalves, M. y Leon, R.E. 1999. Coeficientes de correlación entre medidas in vivo y de la canal en ovinos de la raza Criolla. Congreso Latinoamericano de Especialistas en Pequeños Rumiantes y Camélidos Sudamericanos, 1. Memórias... 239 pp.

Randrianariveloseheno, A.J.M.; Rakotozandriny, J.N.; Hantanirina, I.H.; Razafindratsito, A.N.J.; Razafindraseta, N.N.; Randriamahatana, F. and Rafenomanjato, Z. 2015. Body morphometric of goat local race destined to slaughtering in Madagascar. Global J Anim Sci Res, 3: 271-279.

Resende, K.T.; Medeiros, A.N.; Calegari, A. y Yáñez, E.A. 2001. Utilización de medidas corporales para estimar el peso vivo de caprinos Saanen. Jornadas Científicas Internacionales de la Sociedad Española de Ovinotecnia y Caprinotecnia , 26. Sevilla, España. Memorias... Sociedad Española de Ovinotecniay Caprinotecnia. Sevilla. pp. 340-344.

Ribeiro, M.N.; Gomes Filho, M.A. e Bermejo, J.V.D. 2004. Conservação de raças caprinas nativas do Brasil: Histórico, situação atual e perspectivas. Ribeiro, M.N. Ed. UFRPE, Imprensa Universitária. Recife. 62 pp.

Ribeiro, N.L.; Medeiros, A.N.; Ribeiro, M.N. e Pimenta Filho, E.C. 2004. Estimación del peso vivo de caprinos autóctonos brasileños mediante medidas morfométricas. Arch Zootec. 53: 341-344.

Valdez, C.A.; Fagan, D.V. and Vicera, I.B. 1982. The correlation of body weight to esternal body measurements in goats. Philipp J Anim Ind, 37: 62-89.

Santana, C.J.; Querino, E.C.S.; Costa, F.J.T. e Melo Júnior, U.C. 2000. Manual de caprinocultura. Agronegócios. Sebrae/PE. 43 pp. 
Santana, A.F. De; Costa, G.B. e Fonseca, L.S. 2001. Correlações entre peso e medidas corporais em ovinos jovens da raça Santa Inês. Rev Bras Saúde Prod Anim, 3: 74-77.

SAS. 1999. SAS/STAT User's guide. Version 8. v.2. SAS Institute Inc. Cary. Tadesse, A.; Gebremariam, T. and Gangwar, S.K. 2012. Application of linear body measurements for predicting body weight of
Abergelle goat breed in Tigray region, Northern-Ethiopia. $G J B$ B, 2: 314-319.

Urbano, S.A.; Cândido, E.P.; Lima, C.A.C.; Júnior Dias, W.; Fonseca, F.C.E. e Cavalcanti, F.A.L. 2006. Uso da barimetria para estimar peso corporal de caprinos da raça Canindé. Anais. Congreso Brassileiro de Zootecnia. Recife. 